\title{
TITLE:
}

\section{Why do students participate in medical education?}

\section{$\operatorname{AUTHOR}(\mathrm{S})$ :}

Fujikawa, Hirohisa; Wong, Jeffery; Kurihara, Hiroki; Kitamura, Kiyoshi; Nishigori, Hiroshi

\section{CITATION:}

Fujikawa, Hirohisa ... [et al]. Why do students participate in medical education?. The clinical teacher 2015, 12(1): 46-49

ISSUE DATE:

2015-01-20

URL:

http://hdl.handle.net/2433/197468

RIGHT:

This is an OnlineOpen article. 


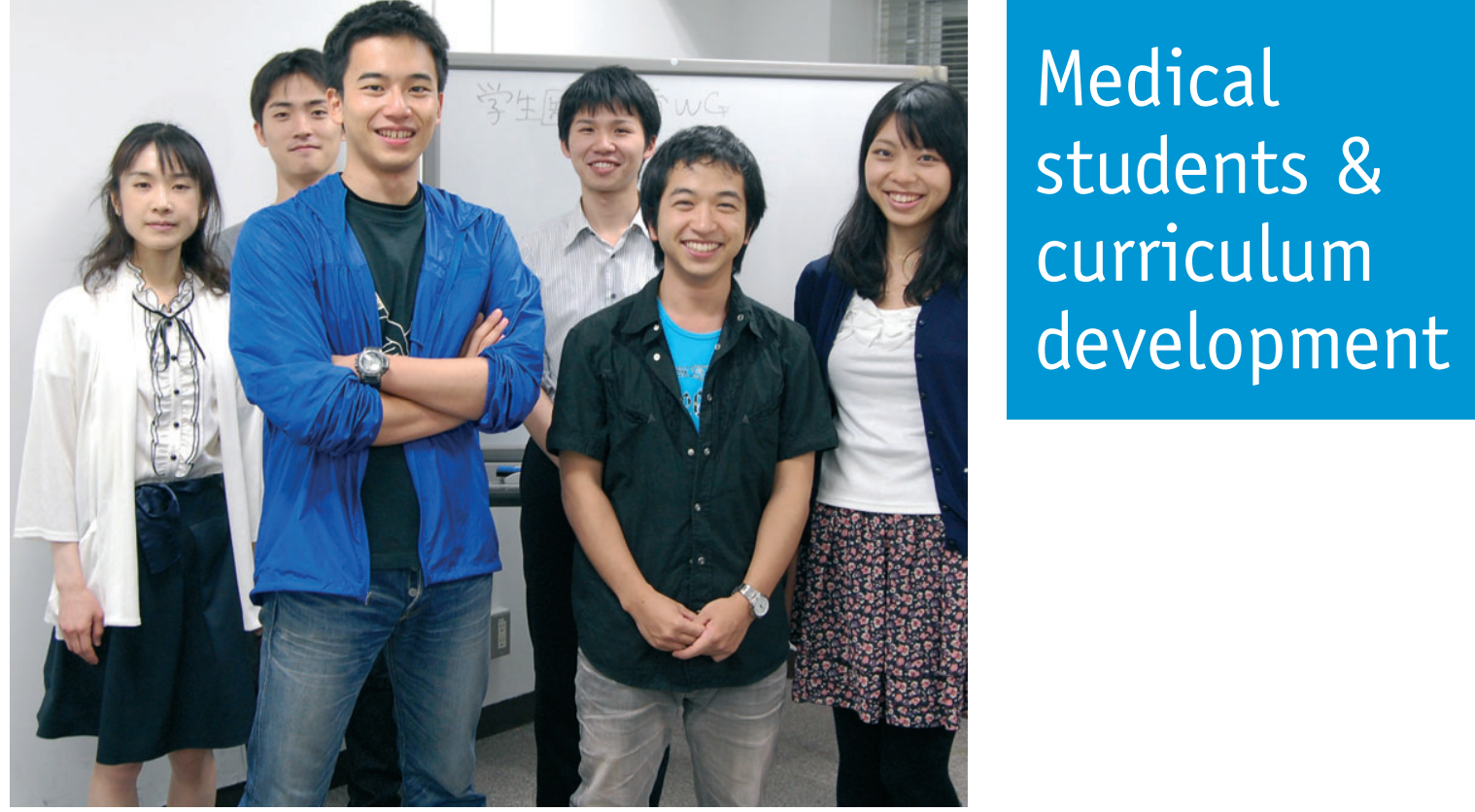

\section{Why do students participate in medical education?}

\section{Hirohisa Fujikawa ${ }^{1}$, Jeffery Wong ${ }^{2}$, Hiroki Kurihara ${ }^{3}$, Kiyoshi Kitamura ${ }^{4}$ and Hiroshi Nishigori ${ }^{5}$}

${ }^{1}$ Department of Medicine, Suwa Central Hospital, Nagano, Japan

2Division of General Internal Medicine \& Geriatrics, Medical University of South Carolina, Charleston, USA

${ }^{3}$ Department of Molecular Cell Biology, University of Tokyo, Japan

4International Research Center for Medical Education, University of Tokyo, Japan

${ }^{5}$ Center for Medical Education, Kyoto University, Japan

\section{What motivates medical students to participate in the process of curriculum development?}

\section{SUMMARY}

Background: Medical student involvement in curriculum development is important; however, little is known about why medical students become engaged in this activity. The aim of this study was to understand what motivates medical students at one university to participate in the process of curriculum development and gain a wider perspective on student engagement in medical education.
Methods: Grounded theory methodology was the foundation of this study. We conducted semi-structured interviews with seven medical students from the University of Tokyo who developed and participated in a group whose aim was to actively contribute towards improving their medical education. The data from the interviews were analysed by thematic synthesis, with triangulation.

Results: Three themes emerged as potential explanations for motivating student behaviour: (1) extracurricular interaction with faculty members; (2) engaging with highly motivated peers; and (3) student values for serving the public.

Conclusions: Students working to improve educational processes at their medical schools had the opportunity to communicate more with faculty members, enjoyed opportunities for networking with other highly motivated peers and enhanced aspects of their developing professionalism. 


\section{INTRODUCTION}

Medical education in Japan has experienced rapid change in recent times. Some of these changes include the institution of formal rotating internships in postgraduate training, beginning in 2004, and the introduction of a nationwide objective structured clinical examination (OSCE) and computer-based test (CBT), beginning in 2005. ${ }^{1}$ In 2013, work began on developing a national accreditation system for undergraduate medical education. These changes have influenced the way that faculty members approach curriculum reform. As an example, medical students have recently become more involved in curriculum development in some, but not all, Japanese medical schools. Furthermore, the importance of student engagement in curriculum development is one of the basic tenets stated in the World Federation of Medical Education (WFME) global standards for quality improvement (World Federation of Medical Education; 2012). ${ }^{2}$

\section{THE UNIVERSITY OF TOKYO STUDENT GROUP FOR MEDICAL EDUCATION}

Previously, student engagement at the University of Tokyo was not the norm because of strained relationships between students and faculty members, stemming from protest incidents in the 1960 s. $^{3}$ After nearly 50 years of little to no involvement in curriculum planning, medical students at the University of Tokyo formed a group whose aim was to actively contribute towards planning and improving medical education at their school, with a little assistance from faculty members at the Centre for Medical Education. The University of Tokyo Student Group for Medical Education (UTSME) comprised between five and 10 volunteer members in total from every year. They met weekly to

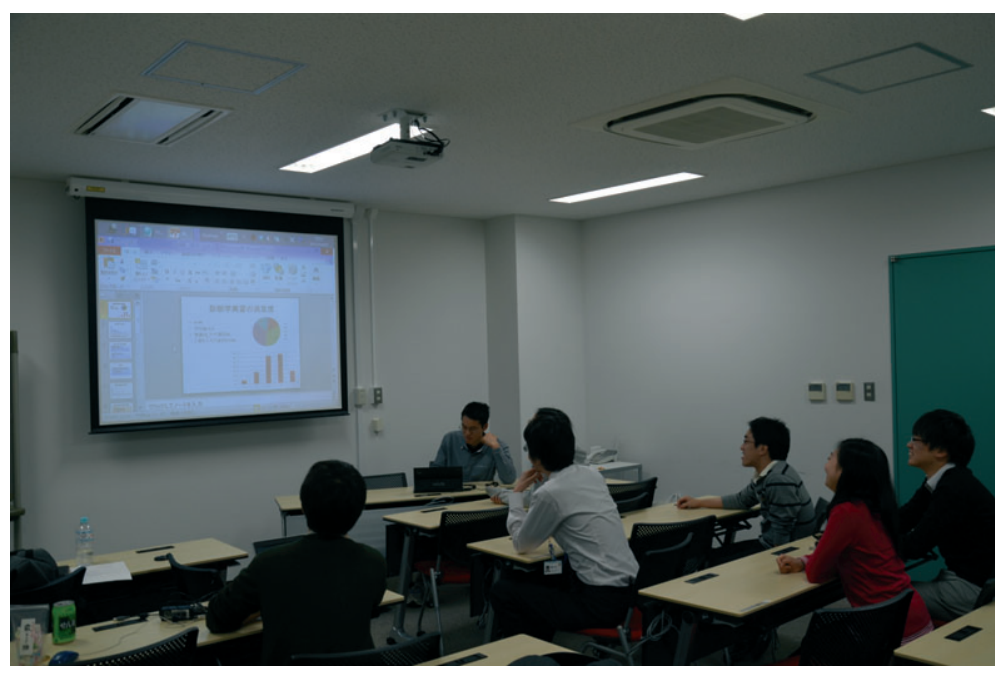

Medical Student

engagement in

curriculum

development

is one of the

basic tenets

stated in the

WFME global

standards
Photo 1. Group discussion in a regular meeting of the University of Tokyo Student working group for Medical Education (UTSME)

discuss issues in medical education (for example, interactivity in teaching and learning) and evaluate the medical school curriculum. Subsequently, they provided formal oral feedback to administrative faculty members in a bimonthly curriculum reform meeting.

\section{AIM OF THIS STUDY}

The UTSME devoted substantial time and effort towards this activity, despite a rather modest impact on the curriculum for a short period. Nevertheless, the students seemed happy and fulfilled by doing this work and we wondered if we could help explain the motivation for their commitment to this activity. In reviewing the literature, little is known about why medical students become engaged in medical education, although their motivation is important to get them involved in the process of improving the curriculum. ${ }^{4}$ We wanted to better understand what motivates students to participate in such activities, especially when there seems to be such little reward for their efforts. Therefore, we crafted this study with the aim of understanding what motivates medical students to participate in refining medical education. Through clarifying this motivation, we expected to gain a wider perspective on student engagement in medical education.

\section{METHODS}

A grounded theory methodology was used for this preliminary qualitative study. ${ }^{5}$ From January to May 2012 at the University of Tokyo, the first author (HF), who was a member of the UTSME, conducted individual semi-structured interviews with all seven members working for the group in 2012 with their consent. The interviews varied in length from 40 to 120 minutes, and the students were asked about what motivated them to participate in the process of medical education reform. All interviews were tape-recorded and transcribed verbatim. The transcriptions were first iteratively read by $\mathrm{HF}$ and then analysed by the thematic synthesis method. In this method, text coding was performed first, followed by the development of descriptive themes and then, in the last stage, analytical themes were generated. ${ }^{6}$ We chose this approach because it is suitable for analysing relatively unstructured, text-based data in an inclusive and rigorous manner. ${ }^{7}$ The last author $(\mathrm{HN})$ read the transcripts independently, and through this triangulation process the identified themes were reconciled to achieve higher reliability in the data analysis. 


\section{Students}

discovered

more about

how faculty

members

think about

education

\section{RESULTS}

Three main themes emerged as potential explanations describing what motivates medical students to participate in the process of medical education reform: (1) extracurricular interaction with faculty members; (2) engaging with highly motivated peers; and (3) student values for serving the public. The students' narratives were serially numbered using the code numbers S1-S7.

\section{Extracurricular interaction with faculty members}

Before developing the group, the students were often fearful of professors and teaching faculty members. This probably arose from limited opportunities for interaction and communication outside of the classroom; however, through working in the group, the students discovered more about how faculty members think about education and some of the inherent difficulties encountered with structuring a learning curriculum. Some representative narratives included:

I enjoyed being able to talk to teachers in the working group because there aren't that many opportunities to talk to professors. I don't think there will be many other opportunities for me to give presentations to them or such either. S1

This might be a bit rude, but I was surprised to know that the teachers devote more thought to our education than I initially realised. I believe that some of the errors in communication between students and teachers may be based on these erroneous assumptions. S2
I started to think that maybe there were more kinds of faculties considering the [educational] matter. S3

\section{Engaging with highly motivated peers}

Many of the student members of the group were somewhat disappointed that their efforts did not result in radical changes in the curriculum or in the educational environment; however, this disappointment seemed offset by the opportunities that were gained by engaging with the highly motivated student colleagues working alongside.

More than anything, I was able to meet many interesting people, like my upperclassmen and classmates, and talk to them. I think that's the biggest gain. 54

What I gained is probably relationships with others. That's all. S5

\section{Things I have gained} from this activity? I feel that there are tons. One is, being able to work with underclassmen, such as... Making such colleagues is definitely huge. The same goes for the upperclassmen, like... s1

\section{Student values for serving the public}

A third theme that seemed to emerge from the transcripts was that members of the group felt an obligation to serve the public. The values subsumed under the concept of noblesse oblige seemed to be displayed through students' voluntary engagement in medical education reforms.

In addition, aren't they doing it ignoring the immediate profit? That is the uniqueness of the members of this group. It is not a study group to learn knowledge that can be immediately put to use. S1

If I consider it a role of 'research' at Tokyo University, I personally don't think it is difficult to find connections. There is a duty to produce human resources that can lead a field, regardless of whether it's practical or fundamental. Therefore, education is important. S2

That is because I have burdened others as much to be educated through the system. I think that progress can only be expected by supporting systems in such manners. S2

\section{DISCUSSION}

In this study, we discovered three main themes that potentially explain why the students at the University of Tokyo chose to devote their time to participate in medical education.

First, we recognised that effective communication between students and faculty members was suboptimal. The fear students in the UTSME felt towards faculty members before becoming involved in the group was noted, and it is likely that even greater fear might be felt by other students. Tutors in Asian (especially Confucian) culture expect a demonstration of respect for the elderly, and these ideals might be a contributing factor. Therefore, allowing students to be engaged in medical education can buffer this relationship and may 
improve the educational environment through enhancing effective communication.

Second, students created a unique network of highly motivated peers through developing the UTSME. It seems paradoxical, but this is probably because little reward for their efforts can be gained through this 'informal' activity. In medical schools that 'formally' tell students to develop these sorts of groups, the students' motivation might be different, although the WFME recommends student engagement in curriculum development.

Lastly, the values of serving the public were found as the third theme for the students' motivation. This might be unique to the University of Tokyo, as it is the oldest university in Japan and has a long-standing tradition of creating leadership in its culture. This concept might be considered tangible manifestations of medical professionalism, and student engagement in medical education can be an opportunity for students to enhance their professionalism, which many medical educators are now struggling to teach. ${ }^{8}$

This study has several limitations. First, we only studied a small number of selected students at a single university in Japan, so the transferability is limited. Second, as the historical past at the University of Tokyo is different than that at other schools, our findings may not accurately reflect other Japanese medical schools. Third, we interviewed students only; the faculty members and administrators, who are likely to have their own perspectives on medical education, were not studied.

\section{CONCLUSIONS}

Students working to improve educational processes at their medical school got the opportunity to communicate more with faculty members, enjoyed engaging with other highly motivated peers and enhanced aspects of their developing professionalism. We hope that the results of this study will enrich the perspectives of both faculty members and students who are involved in students' participation in medical schools.

\section{REFERENCES}

1. Kozu T. Medical Education in Japan. Acad Med 2006;81:1069-1075.

2. World Federation for Medical Education. Quality Improvement in Basic Medical Education. Available at http://www.wfme.org/standards/ bme. Accessed on 23 May 2013.

3. Social Science Japan. Newsletter Available at http://newslet.iss.utokyo.ac.jp/ssj15/ssj15.pdf\#page=3. Accessed on 15 March 1999

4. Bicket M, Misra S, Wright SM, Shochet R. Medical student engagement and leadership within a new learning community. BMC Med Educ 2010;10:20.

5. Lingard $L$, Albert $M$, Levinson $W$. Grounded theory, mixed methods, and action research. BMJ 2008;337:a567.

6. Thomas J, Harden A. Methods for the thematic synthesis of qualitative research in systematic reviews. BMC Med Res Methodol 2008;8:45.

7. Ziebland S, McPherson A. Making sense of qualitative data analysis: an introduction with illustrations from DIPEx (personal experiences of health and illness). Med Educ 2006;40:405-414.

8. O'Sullivan H, van Mook W, Fewtrell $\mathrm{R}$, Wass V. Integrating professionalism into the curriculum: AMEE Guide No. 61. Med Teach 2012;34:e64-e77.
Allowing

students to be

engaged in

medical

education may

improve the

educational

environment

Corresponding author's contact details: Hiroshi Nishigori, Associate Professor, Centre for Medical Education, Kyoto University, Yoshida-konoe cho, Sakyo-ku 606-8500, Japan. E-mail: hiroshi.nishigori@gmail.com

Funding: None.

Conflict of interest: None.

Acknowledgments: The authors wish to thank all the faculties and students who have been involved in medical education reform at the University of Tokyo.

Ethical approval: The Research Ethics Committee at the Graduate School of Medicine and Faculty of Medicine, The University of Tokyo, granted a formal ethical approval for this study [\#2276-(2)].

doi: $10.1111 /$ tct.12240 